\title{
Assessing the ability of human endothelial cells derived from induced-pluripotent stem cells to form functional microvasculature in vivo
}

\author{
Jonathan R. Bezenah ${ }^{1}$ | Ana Y. Rioja ${ }^{2} \mid$ Benjamin Juliar $^{2} \mid$ Nicole Friend $^{2} \mid$ \\ Andrew J. Putnam ${ }^{1,2}$
}

${ }^{1}$ Department of Chemical Engineering, University of Michigan, Ann Arbor, Michigan

${ }^{2}$ Department of Biomedical Engineering, University of Michigan, Ann Arbor, Michigan

\section{Correspondence}

Andrew J. Putnam, PhD, Department of Biomedical Engineering, University of Michigan, 2204 Lurie Biomedical Engineering Building, 1101 Beal Ave, Ann Arbor, 48109 MI

Email: putnam@umich.edu

Funding information

National Institute of General Medical

Sciences, Grant/Award Number:

T32-GM008353; National Heart, Lung, and Blood Institute, Grant/Award Number: R01-HL085339

\begin{abstract}
Forming functional blood vessel networks is a major clinical challenge in the fields of tissue engineering and therapeutic angiogenesis. Cell-based strategies to promote neovascularization have been widely explored, but cell sourcing remains a significant limitation. Induced-pluripotent stem cell-derived endothelial cells (iPSC-ECs) are a promising, potentially autologous, alternative cell source. However, it is unclear whether iPSC-ECs form the same robust microvasculature in vivo documented for other EC sources. In this study, we utilized a well-established in vivo model, in which ECs (iPSC-EC or human umbilical vein endothelial cells [HUVEC]) were coinjected with normal human lung fibroblasts (NHLFs) and a fibrin matrix into the dorsal flank of severe combined immunodeficiency mice to assess their ability to form functional microvasculature. Qualitatively, iPSC-ECs were capable of vessel formation and perfusion and demonstrated similar vessel morphologies to HUVECs. However, quantitatively, iPSC-ECs exhibited a two-fold reduction in vessel density and a three-fold reduction in the number of perfused vessels compared with HUVECs. Further analysis revealed the presence of collagen-IV and $\alpha$-smooth muscle actin were significantly lower around iPSC-EC/NHLF vasculature than in HUVEC/NHLF implants, suggesting reduced vessel maturity. Collectively, these results demonstrate the need for increased iPSC-EC maturation for clinical translation to be realized.

KEYWORDS

endothelial cell, HUVECs, iPSCs, vascularization
\end{abstract}

\section{1 | INTRODUCTION}

Cardiovascular diseases (CVDs) are the leading cause of death worldwide with total yearly health expenditures and costs associated with lost productivity exceeding $\$ 300$ billion and rising (Mozaffarian et al., 2015). Many of these patients suffer from atherosclerosis, characterized by hardening of the vessels and typically caused by buildup of a cholesterol-rich plaque in an artery (Taleb, 2016). Atherosclerotic lesions can cause ischemia, a reduction or obstruction of oxygenated blood supply to tissues, which can lead to tissue damage and eventually necrosis (Davies, 2012). With the number of deaths and costs attributed to CVD expected to rise over the next decade, there is an urgent clinical need for new approaches to revascularize ischemic tissues to prevent necrosis, amputations, and ultimately death (Roger et al., 2011; Tarride et al., 2009).

A variety of therapeutic strategies have been investigated in recent years to direct angiogenesis. Growth factor delivery has been extensively studied to promote endothelial cell (EC) recruitment and eventually increase vessel formation (Kannan et al., 2005; Yancopoulos et al., 2000). However, growth factor based therapies 
face various challenges, including protein instability, the need for precision delivery, and rapid degradation (Sun et al., 2010). Alternative tissue engineering approaches have attempted to revascularize ischemic tissues using cell transplantation (Rubina et al., 2009; Zhang et al., 2008). Codelivering ECs with stromal cells embedded in a hydrogel biomaterial promotes the formation of stable, mature microvasculature (Au, Tam, Fukumura, Jain, 2008; Koike et al., 2004; Melero-Martin et al., 2008; Rouwkema, Rivron, van Blitterswijk, 2008). However, cell-based approaches have their own set of limitations, including potential immunorejection by the host. Perhaps, the most critical challenge that must be overcome is the need for a plentiful cell source to supply the billions of cells required for clinical translation (Ikada, 2006; Saigawa et al., 2004).

A great deal of enthusiasm emerged with the creation of inducedpluripotent stem cells (iPSCs). Using four transcription factors (Oct4, Sox2, Klf4, and cMyc [OSKM]), adult somatic cells can be reprogramed into a pluripotent stem cell-like state (Takahashi \& Yamanaka, 2006). Because iPSCs are dedifferentiated from a potentially autologous somatic cell type, using them as a cell source not only minimizes ethical concerns associated with other pluripotent cell sources but may also potentially bypass immunological concerns in clinical applications (Yee, 2010). Even if derived from allogeneic sources, iPSCs and their theoretical ability to proliferate indefinitely could ultimately overcome biomanufacturing hurdles by providing a large reservoir of cells necessary for human translation (Wong, Sayed, Cooke, 2013).

Endothelial-like cells, characterized by their expression of EC markers, can be differentiated from iPSCs (Ikuno et al., 2017; Yoder, 2015). Their functional abilities to form vessel-like networks both in vitro and in vivo have also been described (Adams et al., 2013; Margariti et al., 2012; Rufaihah et al., 2013). However, because the potential of these cells has been lauded without appropriate benchmarking against other EC sources, we recently investigated the vasculogenic potential of iPSC-ECs in a well-established threedimensional cell culture model of sprouting angiogenesis (Bezenah, Kong, Putnam, 2018). The quantity, quality, and function of the vessellike networks formed by these cells were compared with human umbilical vein endothelial cells (HUVECs), another EC source with a proven capability of capillary morphogenesis. Our worked revealed sprouting by iPSC-ECs was significantly reduced (vs. HUVECs) and identified differences in MMP-9 expression as a possible mechanistic explanation (Bezenah et al., 2018). Despite highlighting these differences, the question remains if this attenuation is only an in vitro phenomenon.

In this present study, we compared the in vivo vascularization potential of iPSC-ECs head-to-head with HUVECs. ECs (either iPSC-ECs or HUVECs) and normal human lung fibroblasts (NHLFs) were coinjected subcutaneously within a fibrin matrix into the dorsal flank of severe combined immunodeficiency (SCID) mice. Vessel formation was characterized by quantifying vessel density, vessel perfusion, and markers of vessel maturity. Our findings demonstrate iPSC-ECs are unable to form vessels of equal quality and quantity compared with an established EC source.

\section{2 | MATERIALS AND METHODS}

\subsection{Cell culture}

HUVECs were harvested from fresh umbilical cords following a previously established protocol (Ghajar et al., 2006). HUVECs were plated with endothelial growth media (EGM-2; Lonza, Walkersville, MD) in tissue culture flasks and cultured at $37^{\circ} \mathrm{C}$ and $5 \% \mathrm{CO}_{2}$. Media were changed every $48 \mathrm{hr}$ and cells were used at passage 3. NHLFs (Lonza) were cultured at $37^{\circ} \mathrm{C}$ and $5 \% \mathrm{CO}_{2}$ in Dulbecco's modified Eagle's media (DMEM; Life Technologies, Grand Island, NY) with 10\% fetal bovine serum (FBS). Culture media were replaced every $48 \mathrm{hr}$ and cells from passage 6 to 10 were used in experiments. iCell ECs (Cellular Dynamics International, Madison, $\mathrm{WI}$ ), referred to as iPSC-ECs, were cultured at $37^{\circ} \mathrm{C}$ and $5 \% \mathrm{CO}_{2}$ in Vasculife VEGF endothelial medium (Lifeline Cell Technology, Fredrick, MD) supplemented with iCell Endothelial Cell Medium Supplement (Cellular Dynamics International), per the manufacturer's instructions. iPSC-EC's tissue culture flasks were coated with $35 \mu \mathrm{g} / \mathrm{ml}$ fibronectin (Invitrogen, Carlsbad, CA) for $1 \mathrm{hr}$ at room temperature before plating the cells. Culture media were replaced every $48 \mathrm{hr}$ and cells from passage 3 were used in experiments.

\section{2 | Sample preparation for subcutaneous injection}

A $2.5 \mathrm{mg} / \mathrm{ml}$ bovine fibrinogen (92\% clottable; Sigma-Aldrich, St. Louis, MO) solution was prepared by dissolving the protein in an appropriate amount of serum-free EGM-2 and placed in a water bath at $37^{\circ} \mathrm{C}$. The solution was sterile filtered through a $0.22 \mu \mathrm{m}$-syringe filter (Millipore, Billerica, MA). Cells were cultured in T-75 flasks to $80 \%$ confluency and rinsed with phosphate-buffered saline (PBS) before being harvested via $0.25 \%$ trypsin incubation for $5 \mathrm{~min}$ at $37^{\circ} \mathrm{C}$ and $5 \% \mathrm{CO}_{2}$. Trypsin was neutralized using DMEM supplemented with $10 \%$ FBS. The cellular suspension was centrifuged ( $200 \mathrm{~g}$ for $5 \mathrm{~min}$ ) and supernatant was aspirated immediately. Cells were resuspended at a 1:1 ratio of ECs:NHLFs in the previously prepared fibrinogen solution at a final concentration of four million cells/ml, totaling two million cells per injection $(500 \mu \mathrm{l})$. Immediately before injection, 5\% FBS and $12 \mu \mathrm{l}$ of thrombin solution ( $50 \mathrm{U} / \mathrm{ml}$; Sigma-Aldrich) were added to the $500 \mu \mathrm{l}$ of fibrinogen-cell solution. A cellular controls containing fibrinogen, FBS, and thrombin were also prepared.

\section{3 | Subcutaneous injections}

All animal procedures were performed in accordance with the National Institutes of Health (NIH) guidelines for laboratory animal usage and approved by the University of Michigan's, Institutional Animal Care and Use Committee. Male CB17/SCID mice (6-8 weeks old; Taconic Labs, Hudson, NY) were used for all experiments. The mice were allowed to acclimate for $\geq 72 \mathrm{hr}$ after arrival. Before surgery, mice were weighed and marked with permanent marker to identify experimental conditions. The mice were then anesthetized in an induction chamber with $5 \%$ isoflurane at $1 \mathrm{~L} / \mathrm{min} \mathrm{O}_{2}$ (Cryogenic 
Gases, Detroit, MI) using a V-1 Tabletop isoflurane vaporizer system equipped with an Active Scavenging Unit (VetEquipt, Livermore, CA). Once mice were fully anesthetized, they were moved to a surgery bench and fitted with an active scavenging nose cone. The isoflurane level was reduced to $1-1.5 \%$, depending on weight, during surgery to maintain anesthesia. Ophthalmic ointment (Puralube ${ }^{\circledR} 499$ vet ointment; Dechra, Overland Park, KS) was added to the eyes of each mouse. An analgesic, carprofen (5 mg/kg), was then administered to each animal via intraperitoneal injection. The dorsal flanks of each mouse were shaved and depilatory agent (Nair; Thermo Fisher Scientific, Pittsburg, PA) was applied to remove any remaining hair. Ethanol and betadine (Thermo Fisher Scientific, Fremont, CA) were applied alternating three times each to sterilize the injection site. The injection samples were then prepared, as described above. Each solution was rapidly mixed and drawn into a $1 \mathrm{~mL}$-syringe fitted with a BD PrecisionGlide ${ }^{\mathrm{TM}} 20 \mathrm{G}$-needle (Becton Dickson, Franklin Lakes, NJ). The mixture $(500 \mu \mathrm{l})$ was immediately injected subcutaneously on the dorsal flank of the mouse, with two implants per animal, one on each flank. The needle was left in the injection site for $1 \mathrm{~min}$ to allow for the solution to polymerize. Mice were then placed in a recovery cage to recover from the anesthesia, and then returned to their normal housing environment. Mice were monitored daily postsurgery. A total of 18 mice ( 4 implants/cell condition $\times 3$ time points $\times 3$ cell conditions $\times 2$ implants/mouse) were used for this study. Bilateral implants were injected per animal, one on each flank in a randomized fashion.

\section{4 | Implant retrieval postprocessing}

Animals were euthanized on Days 4,7 , or 14 after implants were injected. The implants were surgically excised from each mouse via gross dissection and placed immediately in Z-fix (Thermo Fisher Scientific). After a $24 \mathrm{hr}$-fixation, implants were washed thrice in PBS and stored in $70 \%$ ethanol at $4^{\circ} \mathrm{C}$ until further processing. Excess tissue was then removed from the implant using forceps and scissors, and samples were placed in embedding cassettes (UNISETTE cassette with lid; Simport, Beloeli, QC, Canada), dehydrated, and embedded in paraffin using a KD-BMII tissue embedding center (IHC World, Ellicott City, MD). For further analysis, embedded samples were sectioned (6- $\mu \mathrm{m}$ thick sections) with a Thermo Fisher Scientific HM 325 rotary microtome and placed on glass slides with six sections per slide.

\subsection{Hematoxylin and eosin staining}

Paraffin sections on glass slides were dewaxed in xylene baths twice for $5 \mathrm{~min}$. Slides were then washed twice in each decreasing ethanol concentration (100, 95, and 70\%) bath for $3 \mathrm{~min}$. Lastly, slides were rehydrated in deionized water for 3 min before staining with Mayer's hematoxylin (Electron Microscopy Sciences, Hatfield, PA) for 15 min. Slides were then rinsed in tap water for $15 \mathrm{~min}$ and briefly placed in 95\% ethanol for $30 \mathrm{~s}$. Following, slides were immersed in Eosin $Y$ (Sigma-Aldrich) for $1 \mathrm{~min}$ and dehydrated twice in each increasing ethanol concentration (95 and 100\%) bath for $1 \mathrm{~min}$. Samples were cleared by washing in xylene baths twice for $3 \mathrm{~min}$. Toluene mounting solution (Permount; Thermo Fisher Scientific) was added to coverslips before placing on top of each slide. Slides were left to dry before imaging.

\section{6 | Immunohistochemical staining}

Implant region locations were first approximated with the aforementioned hematoxylin and eosin (H\&E) staining. Serial sections were deparaffinized and rehydrated following the same protocol described for hematoxylin and eosin staining. Slides were then steamed in a vegetable steamer $\left(95-99^{\circ} \mathrm{C}\right)$ for $35 \mathrm{~min}$ in an antigen retrieval solution (Dako, Carpinteria, CA) and equilibrated to room temperature for $30 \mathrm{~min}$. Slides were washed thrice in $0.1 \%$ Tween 20 Tris-buffered saline (TBS-T) for $2 \mathrm{~min} /$ wash, whereas changing the baths for every wash. Excess moisture was removed from each slide and the area around the tissue was marked with an ImmEdge pen (Vector Laboratories, Inc, Burlingame, CA). The Dako EnVision System-HRP (DAB) kit (Dako) was utilized to detect labeled antigens in the sections. First, peroxidase blocking solution was added to each tissue section for $5 \mathrm{~min}$. Slides were subsequently washed thrice in $0.1 \%$ Tween 20 TBS-T for $2 \mathrm{~min} /$ wash (changing baths between washes). Mouse anti-human CD31 primary antibody (1:50; Dako), mouse anti-human smooth muscle actin antibody (1 A4 (asm-1)); (1:200; Thermo Fisher Scientific), or mouse anti-human collagen-IV (COL-IV) monoclonal antibody (COL-94; 1:500; Thermo Fisher Scientific) were diluted in TBS-T and added to each tissue sample. Primary antibodies were incubated for $16 \mathrm{hr}$ at $4^{\circ} \mathrm{C}$ for both CD31 and COL-IV and $2 \mathrm{hr}$ at room temperature for $\alpha$-smooth muscle actin ( $\alpha$-SMA). After incubations, samples were rinsed thrice with TBS-T at $2 \mathrm{~min} / \mathrm{wash}$. The horseradish peroxidase-labeled polymer solution was then added to each sample and incubated for $30 \mathrm{~min}$ at room temperature. Samples were washed once again as described above. DAB + substrate-chromogen buffer solution was then added to each tissue section for $5 \mathrm{~min}$, and immediately rinsed in deionized water for 30 s. Samples were counterstained with hematoxylin for $15 \mathrm{~min}$, followed by a $15 \mathrm{~min}$ wash in tap water. Slides were then washed, as described for H\&E staining, with $95 \%$ ethanol, $100 \%$ ethanol, and xylene to dehydrate the samples. Lastly, toluene mounting solution was added to each slide before covering the samples with coverslips. Slides were left to dry before imaging.

\section{7 | Vessel quantification}

After staining, slides were imaged using an Olympus IX81 microscope with a DP2-Twain color camera (Olympus America, Center Valley, PA) and the CellSens Imagining Software (Olympus) for visualizing stained slides. Brightfield images were taken using $x 4$ and $x 20$ objectives for each of the various positively-stained marker region of the sections. A x40 objective was used to image individual vessels. Positively-stained sections from three separate implants for each condition, with five random $\times 20$ images per section, were then quantified to determine average vessel density on a per $\mathrm{mm}^{2}$ basis for each of the indicated expression markers. Structures were considered a blood vessel if they exhibited a hollow lumen surrounded by a complete brown rim of positive stain. Quantification 
of vessels derived from the transplanted human cells was conducted by two independent evaluators via a one-side blinded study for hCD31. Lumen diameter was also quantified by each evaluator using Image J (National Institutes of Health, Bethesda, MD). Quantification of vessels expressing $\alpha$-SMA and COL-IV proceeded via the same methods described above, but were unblinded. Acellular fibrin controls were not analyzed or shown because they did not contain any human EC-derived vessels.

\section{8 | Statistical analysis}

Statistical analyses were performed using the StatPlus (AnalystSoft Inc, Walnut, CA). One-way analysis of variance with a Bonferroni posttest was used to assess statistical significance between datasets. Data are reported as mean \pm standard error of mean (SEM). Statistical significance was assumed when $p<0.05$.

\section{3 | RESULTS}

\section{1 | iPSC-EC/NHLF fibrin implants are capable of vascular morphogenesis in vivo}

In this study, HUVECs and iPSC-derived ECs were characterized for their abilities to form microvasculature when coinjected with NHLFs in a three-dimensional fibrin matrix into subcutaneous pockets in vivo. H\&E staining of tissue sections from these implants demonstrated the presence of vessel structures across all time points
(Figure 1). Despite the evidence of vessel formation and the presence of lumens for both EC types at each time point, the vessels formed by Day 4 were comparatively smaller relative to the later time points (Figure 1a,d). Vessels identified in Day 4 sections also showed minimal evidence of inosculation with host microvasculature due to relative absence of host erythrocytes observed within the lumens or in the surrounding matrix. However, by Days 7 and 14, constructs exhibited larger vessel morphologies and host erythrocytes were increasingly apparent throughout the implant region (Figure 1b,c,e, and f). Sections from both Days 7 and 14 show evidence of vessel perfusion. The erythrocytes were largely contained within the lumens of the neovessels formed by both EC types (as opposed to leaking into the interstitial tissue space) at the Day 14 time point. Collectively, these results suggest the iPSC-ECs and HUVECs form functional microvasculature with similar qualities in vivo.

\section{2 iPSC-ECs produce vessels with comparable morphologies and similar diameters}

To validate the observations from the $H \& E$-stained sections and confirm the human origins of the neovasculature, human ECs were identified in explanted tissue constructs via immunohistochemical staining of human CD31 (Figure 2a-f). Both EC types demonstrated hollow lumens surrounded by a brown rim of positive staining at each time point, confirming successful vessel formation by the implanted human cells as suggested by the H\&E stain. Vessels also demonstrated morphological similarities with both cell conditions. Furthermore,
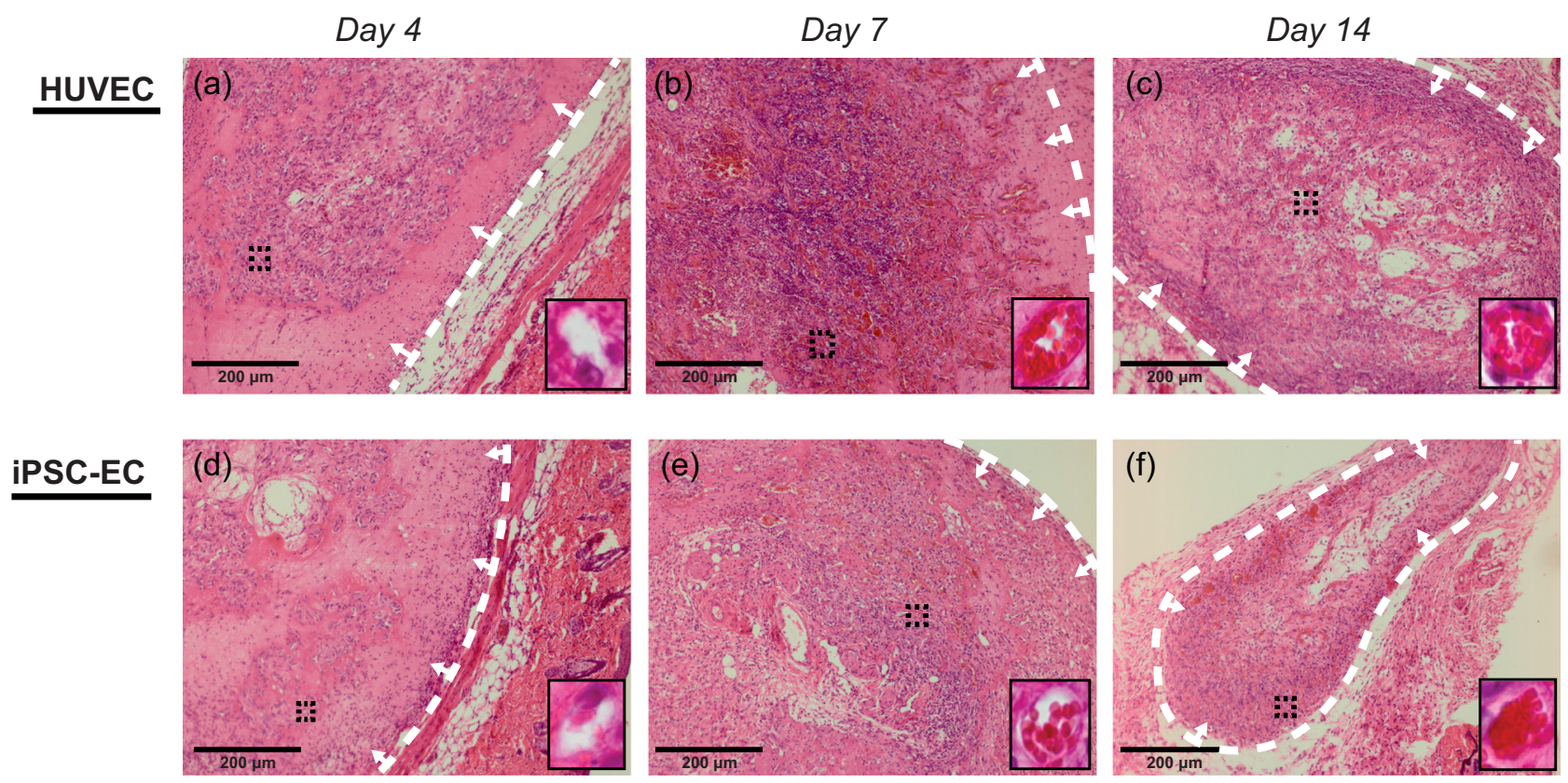

FIGURE 1 Histological staining illustrates in vivo vessel formation and similar phenotypes across cell types representative images of subcutaneous implants stained with hematoxylin and eosin. Implants were formed by coinjecting HUVECs (a-c) or iPSC-ECs (c-f) with NHLFs in $2.5 \mathrm{mg} / \mathrm{ml}$ fibrin. Implants were harvested at Days 4 (a,d), 7 (b,e), and 14 (c,f). Insets are images from a x40 objective lens, taken from the region indicated with black-dashed lines, to more clearly show vessel morphologies and the presence of host erythrocytes. The white-dashed lines are to indicate the boundary between the implant and mouse tissue, whereas arrows point to the implant region (Scale bar $=200 \mu \mathrm{m})$. HUVEC: human umbilical vein endothelial cell; iPSC-EC: induced-pluripotent stem cell-derived endothelial cell [Color figure can be viewed at wileyonlinelibrary.com] 
Day 4

HUVEC

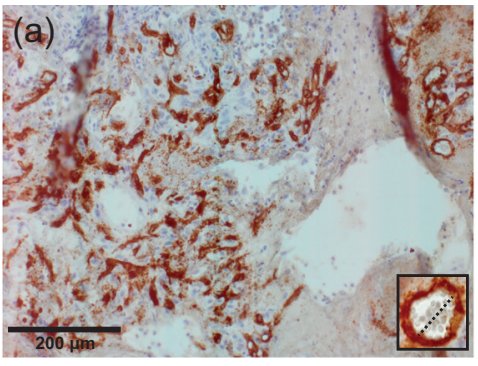

Day 7

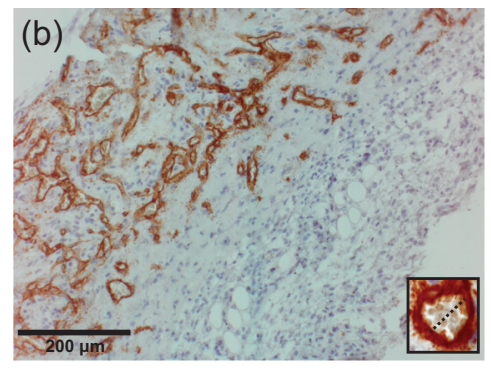

Day 14

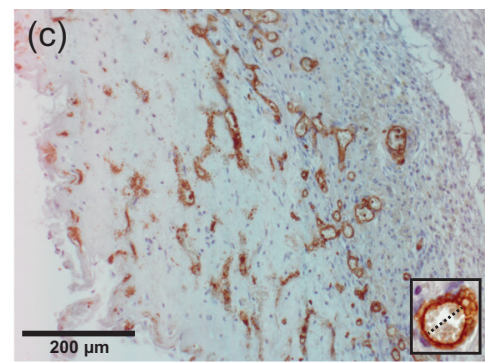

iPSC-EC
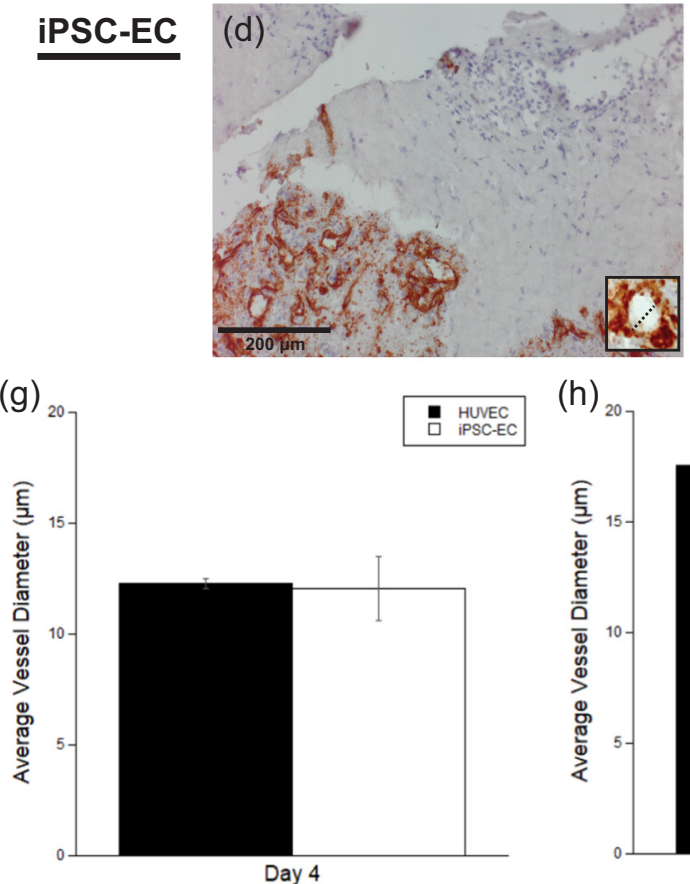

(h)

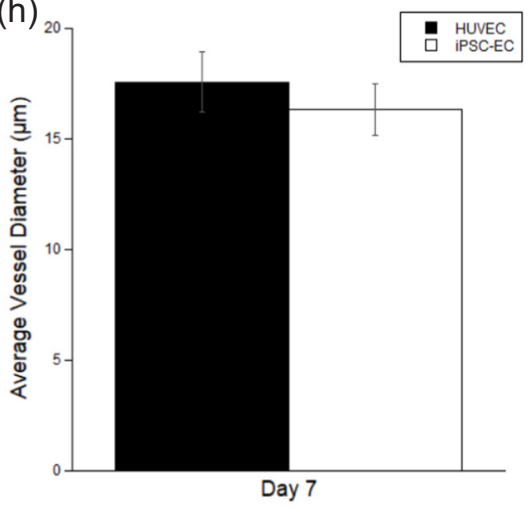

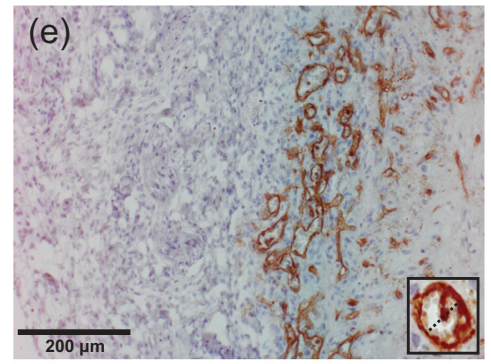

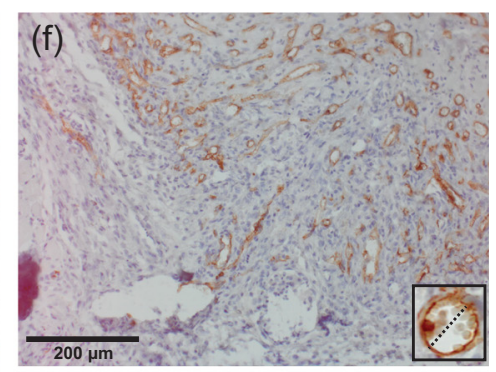

(i)

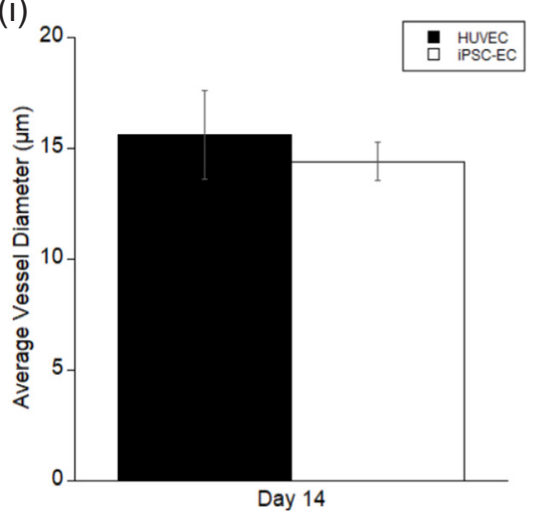

FIGURE 2 Both iPSC-ECs and HUVECs express comparable vessel morphologies with similar vessel diameters representative images of human CD31-stained HUVECs $(a-c)$ or iPSC-ECs (d-f) subcutaneous implants at various time points. All images were counterstained with hematoxylin. Implants were harvested at Days $4(a, d), 7$ (b,e), and 14 (c,f). Insets are images from a x40 objective lens and dashed lines are present to more clearly show similarities between vessel diameters (Scale bar $=200 \mu \mathrm{m}$ ). Vessel diameters, both with or without erythrocytes, were measured and quantified over a total of 5 random images per selected section across three separate animals. Quantifications were single-blinded and averaged at the given time points (g-i). Error bars indicate \pm SEM. HUVEC: human umbilical vein endothelial cell; iPSC-EC: induced-pluripotent stem cell-derived endothelial cell; SEM: standard error of mean [Color figure can be viewed at wileyonlinelibrary.com]

vessel lumens demonstrated similarities in size. Quantification of the inner lumen diameter supported these qualitative observations (Figure 2g-i) demonstrating no significant differences between the HUVEC and $\mathrm{IPSC}-\mathrm{EC}$ condition across all time points $(12.03 \pm 1.45$, $16.33 \pm 1.19$, and $14.04 \pm 1.15 \mu \mathrm{m}$ for iPSC-ECs vs. $12.30 \pm 0.38$, $17.58 \pm 1.32$, and $15.59 \pm 2.01 \mu \mathrm{m}$ for HUVECs on Dayds 4,7 , and 14 , respectively). Despite slightly smaller lumens at the Day 4 time point, there were also no significant differences between time points for each of the cell conditions (Figure 2j,k).

\section{3 | iPSC-ECs exhibit deficiencies in vessel formation compared to HUVECs in vivo}

Our previous study demonstrated that iPSC-ECs are quantitatively deficient in vascular network formation in vitro compared with
HUVECs (Bezenah et al., 2018). To investigate the extent of network formation in vivo, sections from subcutaneous implants were stained with an EC marker, hCD31, and the number of vessels throughout a given area were quantified. Vessels with clearly identifiable lumens surrounded by a positive hCD31 brown rim were counted. Representative images of average vessel density for both HUVECs and iPSCECs at varying time points are shown in Figure 3a-f. Quantification of the vessel density (Figure $3 \mathrm{~g}-\mathrm{i}$ ) revealed significant differences in the average number of vessels $/ \mathrm{mm}^{2}$ at Days 4 and 7 (42.27 \pm 4.82 and $86.43 \pm 21.86 \mu \mathrm{m}$ for HUVECs vs. $25.48 \pm 4.41$ and $35.58 \pm 7.62 \mu \mathrm{m}$ for iPSC-ECs on Days 4 and 7, respectively). However, at later time points, Day 14, there were no significant differences between the IPSC-EC and HUVEC condition with relatively equal average number of vessels $/ \mathrm{mm}^{2}$ (37.61 $\pm 13.61 \mu \mathrm{m}$ for HUVECs vs. $36.46 \pm 3.76 \mu \mathrm{m}$ for iPSC-ECs on Day 14, respectively). Vessel density peaked for the 


\section{Day 4}

HUVEC

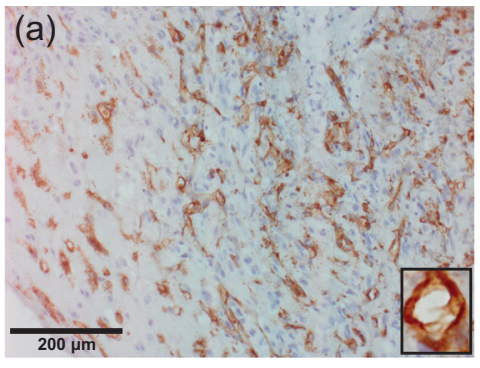

IPSC-EC

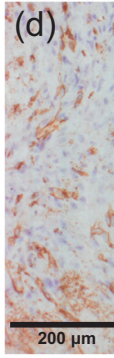

(g)

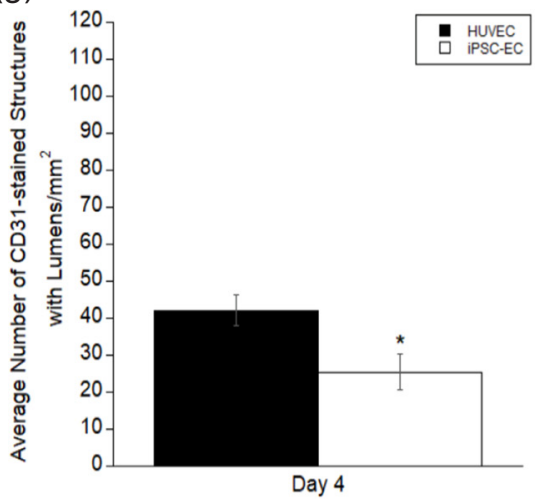

(h)
Day 7
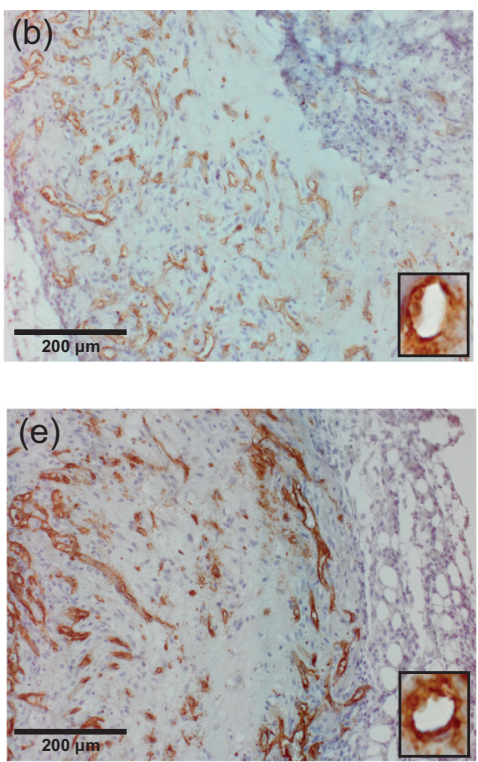

(i)
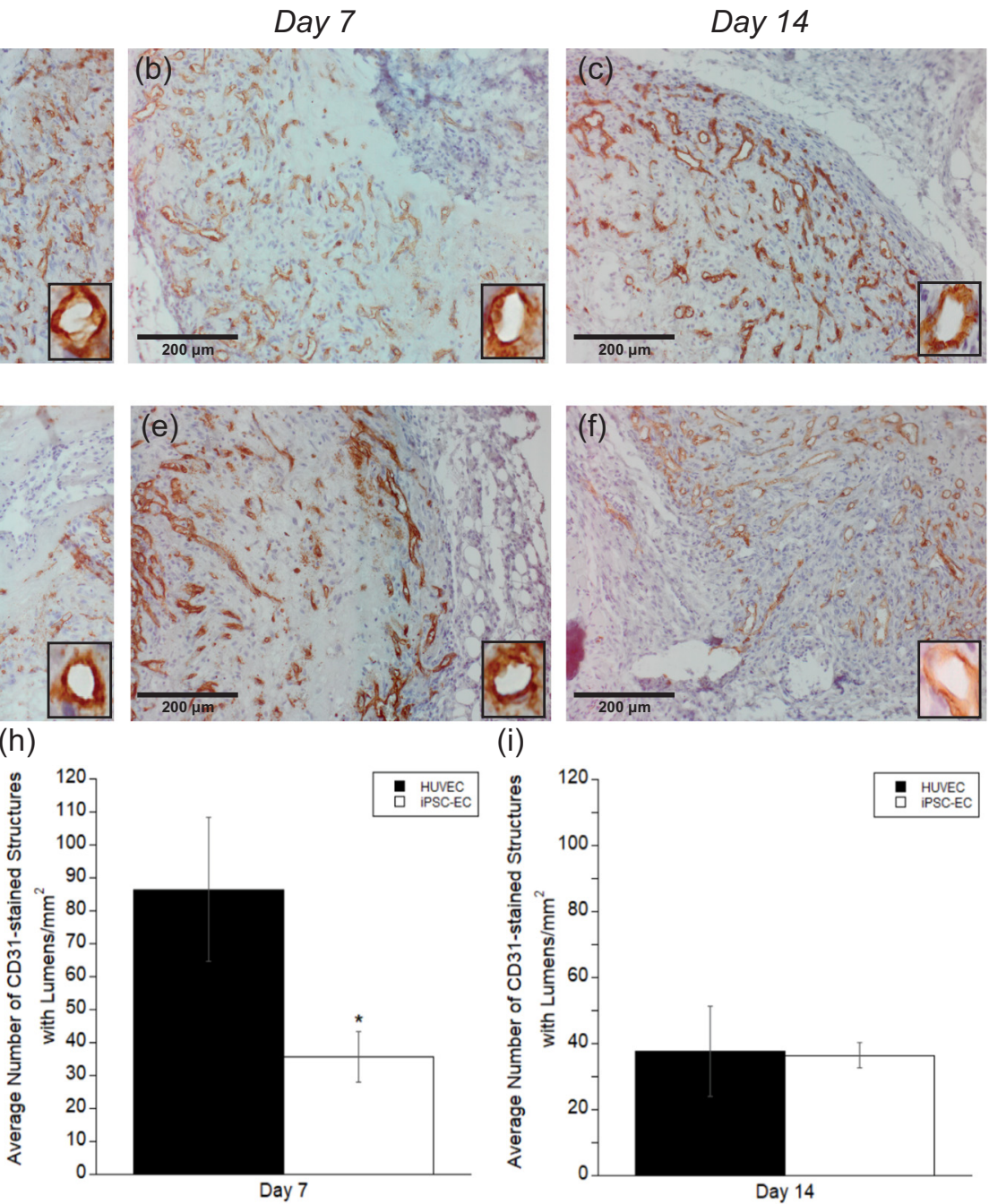

FIGURE 3 iPSC-ECs exhibit deficiencies in vessel lumen formation compared with HUVECs representative images of subcutaneous implants immunohistochemically stained for human CD31 demonstrate the formation of vessel structures with lumens by HUVECs (a-c) or iPSC-ECs $(d-f)$ in subcutaneous implants at various time points. All images were counterstained with hematoxylin. Implants were harvested at Days 4 $(\mathrm{a}, \mathrm{d}), 7$ (b,e), and 14 (c,f). Insets are images from a $x 40$ objective lens to more clearly show vessel lumens (Scale bar $=200 \mu \mathrm{m})$. A total of five random images per selected section across three separate animals were quantified for $+h C D 31$ vessels with lumens. Quantifications were single-blinded, averaged, and normalized to the respective, +hCD31 implant area of each EC type at the given time points (g-i). ${ }^{*} p<0.05$ and comparing the indicated condition to the HUVEC condition. Error bars indicate \pm SEM. HUVEC: human umbilical vein endothelial cell; iPSC-EC: induced-pluripotent stem cell-derived endothelial cell; SEM: standard error of mean [Color figure can be viewed at wileyonlinelibrary.com]

HUVECs at Day 7 and regressed by Day 14, whereas iPSC-EC vessel density increased over time.

\subsection{I iPSC-ECs vessels exhibit less perfusion compared to HUVECs}

Once the baseline density of vessels formed by the implanted cell types was established, any differences in functionality of these newly formed vessels were examined. To evaluate functional anastomoses with the host vasculature, the numbers of vessel lumens perfused with erythrocytes were quantified. Once again, vessels were quantified only if lumens surrounded by a positive hCD31 brown rim were clearly present and erythrocytes were clearly visible within the lumen.
Representative images of average number of perfused vessels for both HUVECs and iPSC-ECs at varying time points are shown (Figure 4a-f). Erythrocytes can clearly be seen in higher magnification inserts. Quantification of these vessels (Figure 4g-i) demonstrated no significant differences in the average number of perfused vessels/ $\mathrm{mm}^{2}$ at Day $4(4.54 \pm 1.89 \mu \mathrm{m}$ for iPSC-ECs vs. $2.39 \pm 2.25 \mu \mathrm{m}$ for HUVECs). However, at Day 7, iPSC-ECs exhibited a statistical significant reduction in perfused vessel density compared with HUVECs $(22.83 \pm 4.06 \mu \mathrm{m}$ vs. $72.81 \pm 21.72$, respectively). No significant differences were seen once again by Day 14 . Whereas iPSC-EC vessel perfusion increased over time, HUVEC vessel perfusion peaked at Day 7, but decreased to similar levels as iPSCECs by Day 14. 
Day 4

HUVEC
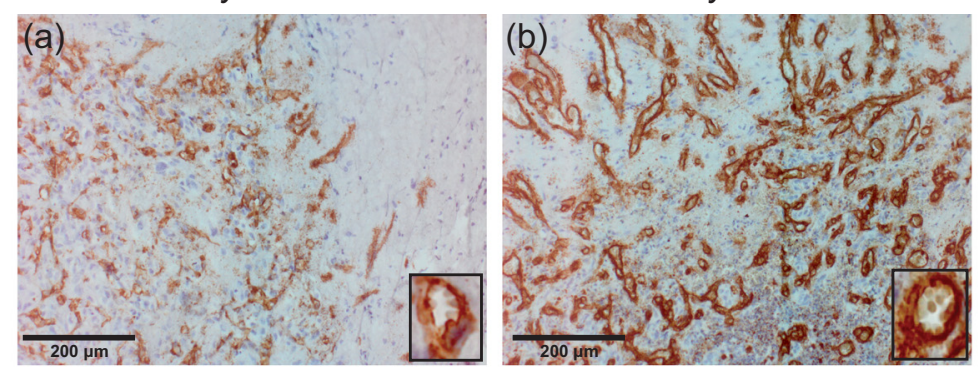

Day 7

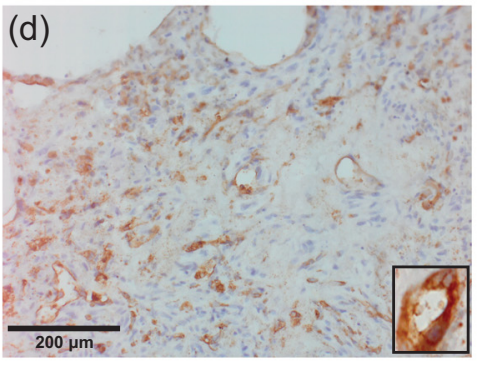

(h)

(g)

iPSC-EC

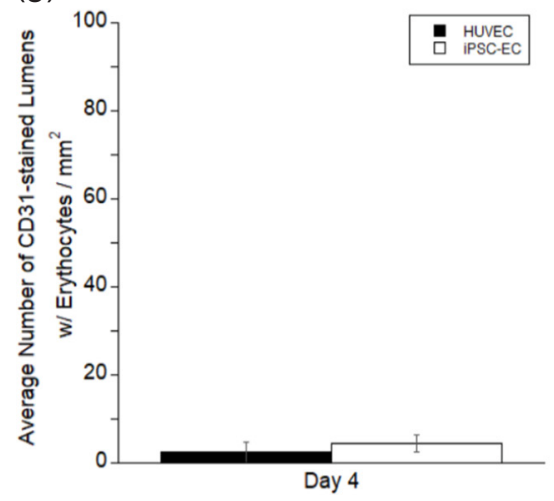

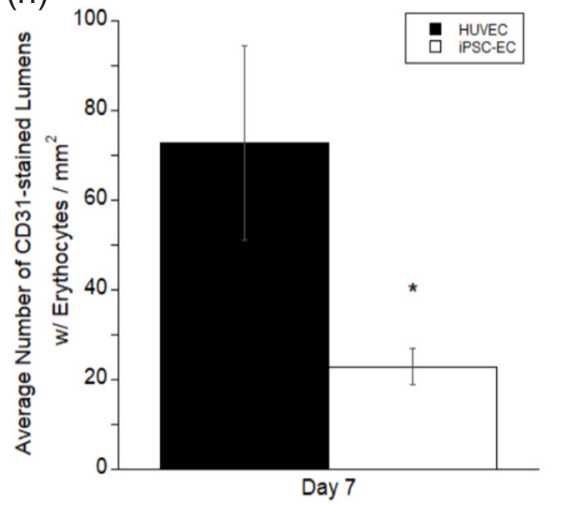

Day 14
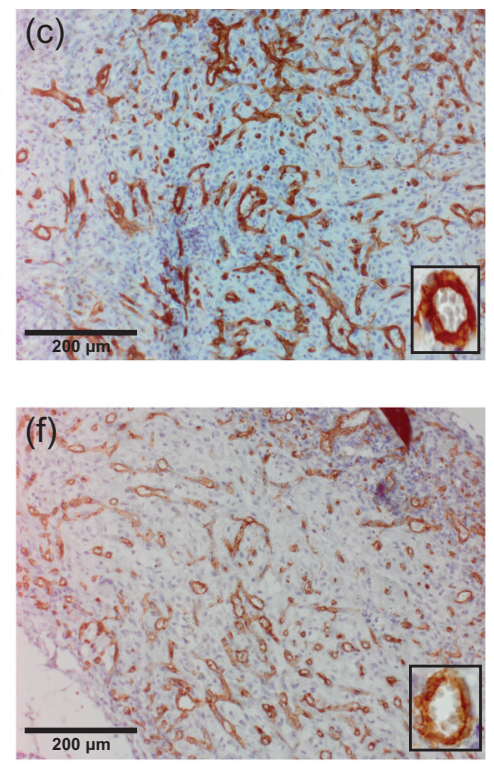

(i)

FIGURE 4 iPSC-ECs vessels are less perfused compared with those formed by HUVECs representative images of subcutaneous implants immunohistochemically stained for human CD31 demonstrate the formation of vessel structures with perfused lumens (identified by the presence of erythrocytes) by HUVECs (a-c) or iPSC-ECs ( $d-f)$ in subcutaneous implants at various time points. All images were counterstained with hematoxylin. Implants were harvested at Days 4 (a,d), 7 (b,e), and 14 (c,f). Insets are images from a x40 objective lens to more clearly show vessel lumens (Scale bar $=200 \mu \mathrm{m}$ ). A total of five random images per selected section across three separate animals were quantified for $+h C D 31$ vessels with erythrocytes in the lumens. Quantifications were single-blinded, averaged, and normalized to the respective +hCD31 implant area of each EC type at the given time points $(\mathrm{g}-\mathrm{i}) .{ }^{*} p<0.05$ and comparing the indicated condition to the HUVEC condition. Error bars indicate \pm SEM. HUVEC: human umbilical vein endothelial cell; iPSC-EC: induced-pluripotent stem cell-derived endothelial cell; SEM: standard error of mean [Color figure can be viewed at wileyonlinelibrary.com]

\section{5 iPSC-ECs express differences in vessel maturity in vivo compared to HUVECs}

Despite the iPSC-ECs' ability to form vessel-like structures in vivo, the quality of the structures was also examined to determine if they exhibit qualitative characteristics of mature capillaries in vivo. To assess vessel development and maturity, sections serial to the positively-stained hCD31 samples in the previous experiments were immunohistochemical (IHC) stained and quantified for various maturity markers to identify pericytic association of the coimplanted NHLFs and basement membrane deposition by the ECs. Vessels were quantified only if they were previously identified as human origin through hCD31 staining in former tissue section, and erythrocytes were present in the lumen. Representative images reveal the presence of $\alpha$-SMA, across all time points for both iPSC-ECs and HUVECs, suggesting recruitment and a pericyte-like phenotype of the coimplanted NHLFs (Figure 5a-d). Pericytes stabilize nascent endothelium and are characterized by physical association with ECs as well as expression of molecular markers such as $\alpha$-SMA (Skalli et al., 1989). Quantification of the vessel density indicated significant differences in $\alpha$-SMA at both time points between iPSC-ECs and HUVECs (Figure $5 \mathrm{e}, \mathrm{f}$ ). The number of vessels surrounded by $\alpha$-SMA also increased from Days 7 to 14 for both cell conditions.

We next characterized basement membrane deposition with human collagen-IV immunohistochemical staining. COL-IV is a prominent 
Day 7
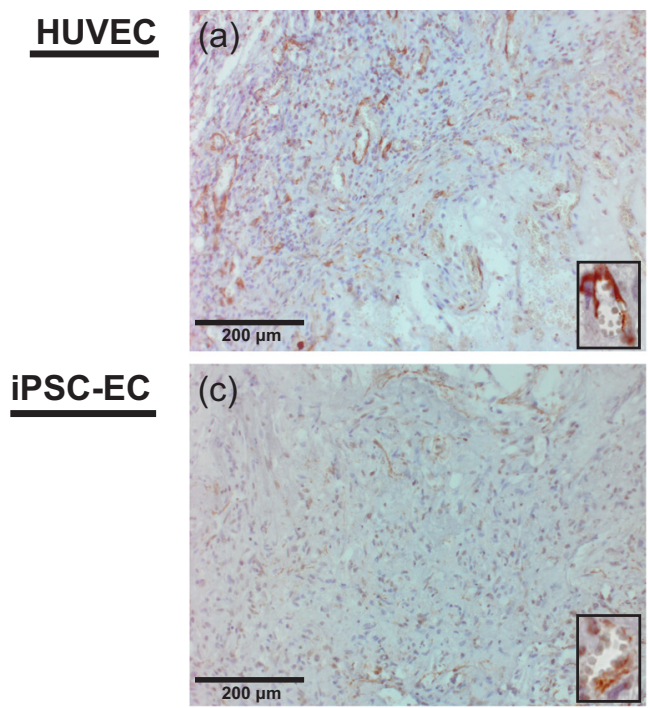

(e)
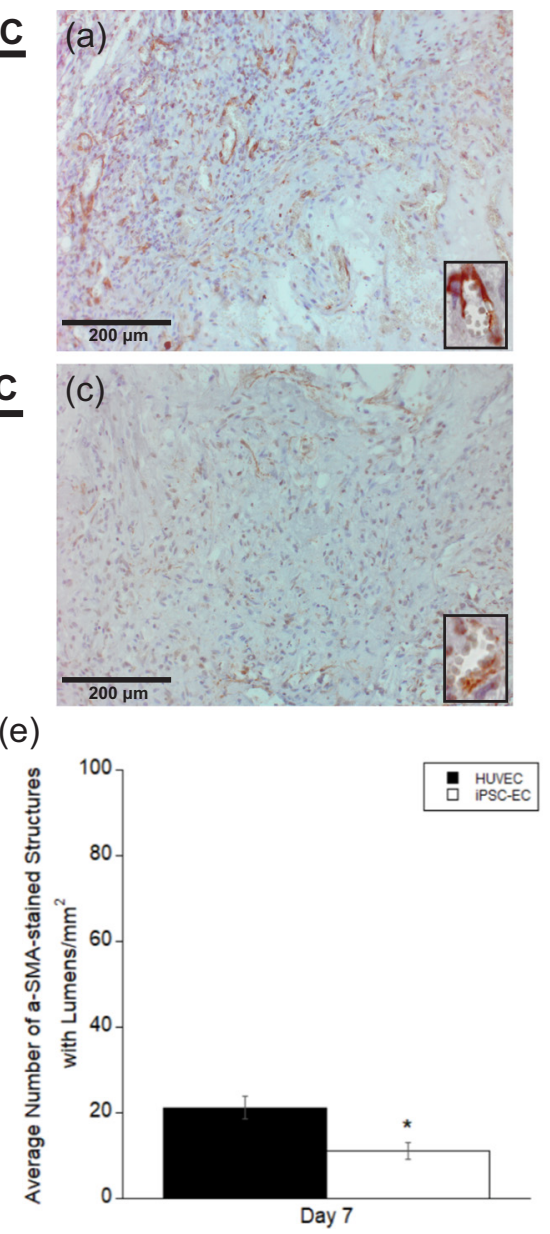

Day 14
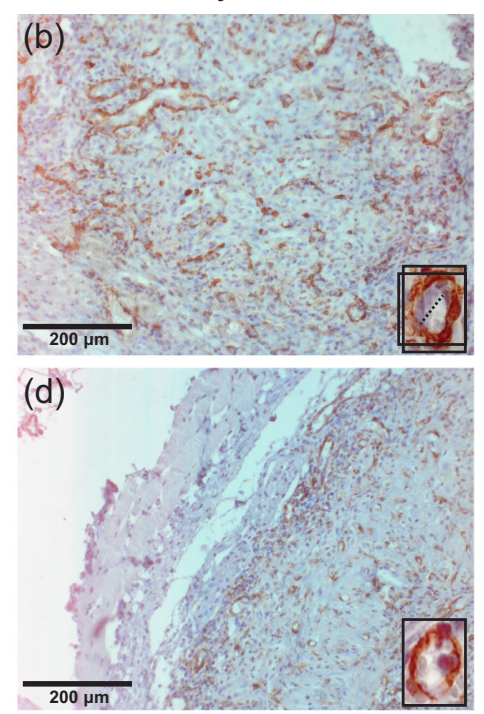

(f)

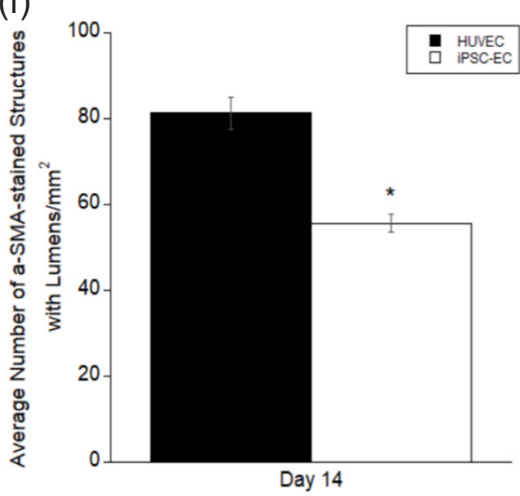

FIGURE $5 \alpha$-SMA staining of vessels formed by iPSC-ECs show differences in vessel maturity compared with those formed by HUVECs representative images of $\alpha$-SMA-stained histological sections from subcutaneous implants formed from either HUVECs (a,b) or iPSC-ECs (c,d) at various time points. All images were counterstained with hematoxylin. Implants were harvested at Days 7 (a,c) and 14 (b,d). Insets are images from a $\times 40$ objective lens to more clearly show positive $\alpha$-SMA staining around vessel lumens (Scale bar $=200 \mu \mathrm{m}$ ). Vessel lumens surrounded by $\alpha$-SMA were quantified over a total of five random images per selected section across three separate animals. Quantifications were averaged and normalized to the respective +human $\alpha$-SMA implant area of each EC type at the given time points (e,f). ${ }^{*} p<0.05$ and comparing the indicated condition to the HUVEC condition. Error bars indicate \pm SEM. HUVEC: human umbilical vein endothelial cell; iPSC-EC: induced-pluripotent stem cell-derived endothelial cell; SEM: standard error of mean; $\alpha$-SMA: $\alpha$-smooth muscle actin [Color figure can be viewed at wileyonlinelibrary.com]

component in basement membranes (Li and Thompson, 2003). Representative images reveal the presence of COL-IV across all time points for both iPSC-ECs and HUVECs (Figure 6a-d). Quantification of the vessel density indicated significant differences in COL-IV at both time points between iPSC-ECs and HUVECs (Figure 6e,f). However, there was little change in expression between the two time points for both cell conditions. Collectively, this data demonstrates that are iPSCECs vessels lack maturity in comparison with HUVEC.

\section{DISCUSSION}

Due to a critical need for a more sustainable and abundant clinically relevant cell source, the vascularization potential of iPSC-ECs is of increasing interest. In previous studies, using a three-dimensional fibrin-based in vitro model, we demonstrated that iPSC-ECs' capillary morphogenesis was significantly attenuated, with reduced MMP-9 expression as one possible candidate for such phenomena (Bezenah et al., 2018). Whereas prior studies have shown that iPSC-ECs are capable of forming vessel-like structures in vivo within supporting Matrigel matrices (Adams et al., 2013; Margariti et al., 2012), there is little evidence comparing their in vivo potential with more widely utilized EC sources. Therefore, this study explored the ability of iPSCECs to create functional vessel-like structures in a fibrin-based subcutaneous implant and compared the quantity and quality of such vessels formed against HUVECs. When codelivered with stromal fibroblasts (NHLFs), iPSC-ECs formed functional microvessels that inosculated with host vasculature; however, the resultant vasculature was of significantly lower density quantitatively and less mature qualitatively when compared to that formed by HUVECs.

Fibrin was selected as the material for implantation because it is a naturally occurring biopolymer that promotes wound healing and 
Day 7

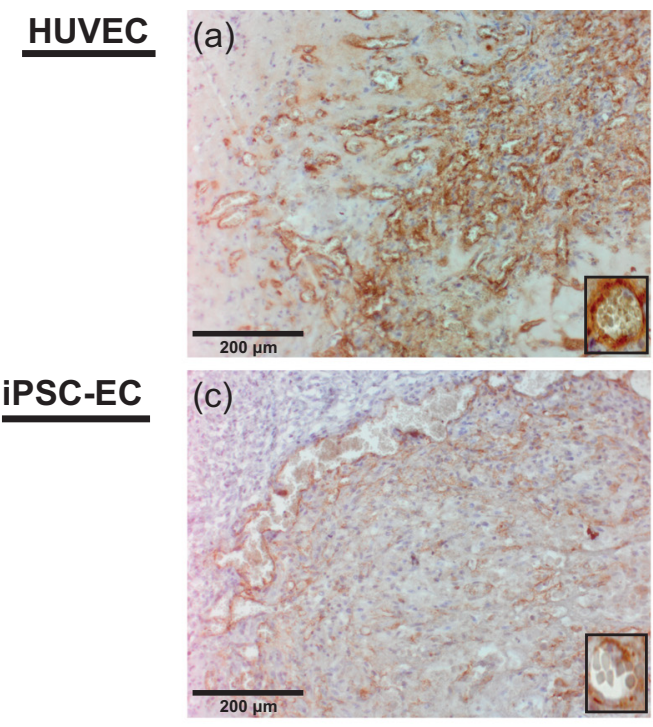

(e)

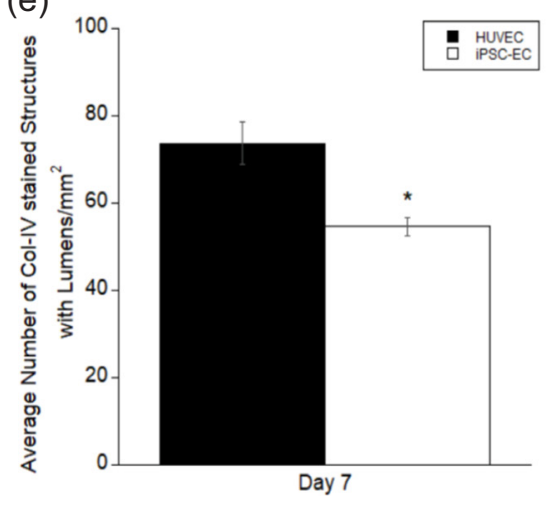

Day 14
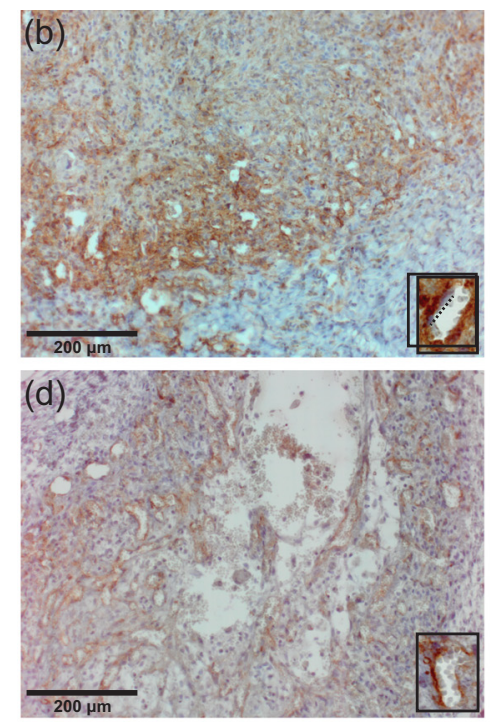

(f)

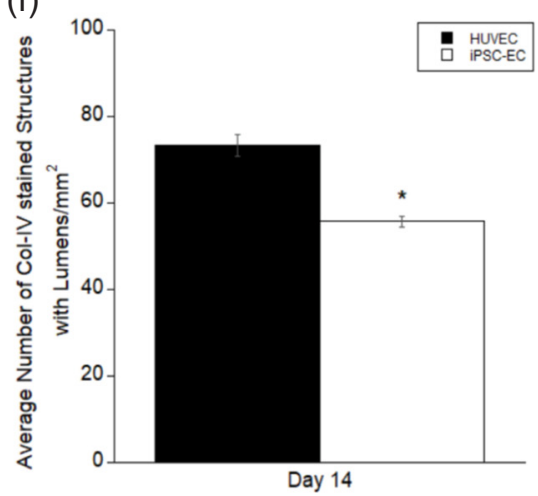

FIGURE 6 COL-IV staining of vessels formed by iPSC-ECS show differences in vessel maturity compared with those formed by HUVECs representative images of COL-IV-stained histological sections from subcutaneous implants formed from either HUVECs (a,b) or iPSC-ECs (c,d) at various time points. All images were counterstained with hematoxylin. Implants were harvested at Days 7 (a,c) and 14 (b,d). Insets are images from a $\times 40$ objective lens to more clearly show COL-IV surrounding vessel lumens (Scale bar $=200 \mu \mathrm{m}$ ). Vessel lumens surrounded by COL-IV were quantified over a total of five random images per selected section across three separate animals. Quantifications were averaged and normalized to the respective +human COL-IV implant area of each EC type at the given time points (e,f). ${ }^{*} p<0.05$ and comparing the indicated condition to the HUVEC condition. Error bars indicate \pm SEM. HUVEC: human umbilical vein endothelial cell; iPSC-EC: inducedpluripotent stem cell-derived endothelial cell; SEM: standard error of mean [Color figure can be viewed at wileyonlinelibrary.com]

neovascularization (Christman et al., 2004) and is Food and Drug Administration cleared for some uses in humans (Ceccarelli \& Putnam, 2014). In addition, fibrin was selected for these studies due to its track record of supporting neovascularization in vivo (Blache \& Ehrbar, 2018; Ceccarelli \& Putnam, 2014; Christman et al., 2004). and to match our prior in vitro study in which we evaluated the potential of iPSC-ECs relative to HUVECs (Bezenah et al., 2018). NHLFs were chosen due to their ability to aid in the formation of microvascular networks, adopt a mural cell-like localization around the vessel-like structures, and express a subset of pericyte markers as previously reported (Ghajar et al., 2006; Ghajar et al., 2008; Grainger, Carrion, Ceccarelli, Putnam, 2013; Peterson et al., 2014). We selected a 1:1 ratio of ECs to stromal cells based on previous studies from our group and others (Grainger et al., 2013; Melero-Martin et al., 2008; Rao et al., 2012). HUVECs are a robust EC source and were selected for their proven capability of capillary morphogenesis not only in the model used for this study, but in other in vivo models as well (D.Y. Chen et al., 2004; Grainger et al., 2013; Kniazeva, Kachgal, Putnam, 2011). The animal model used here has been widely explored in the literature to approximate wound healing and test the ability of transplanted human cells to form vasculature (Allen, Melero-Martin, Bischoff, 2011; X. Chen et al., 2010; Melero-Martin et al., 2008; Traktuev et al., 2009) This model also avoids potential immunorejection of the human cells injected into SCID mice.

$H \& E$ staining of the retrieved implants suggested some similarities, in terms of morphology and phenotype, across the different EC conditions. Both iPSC-ECs and HUVECs formed vessels with similar lumen diameters and shapes across all time points. Capillary diameters are typically around $4 \mu \mathrm{m}$ (Potter \& Groom, 1983). Whereas our results reveal three- to four-fold larger lumen 
diameters, capillary diameters can range much larger depending on the tissue (Wiedeman, 1963). The larger diameters could also indicate less mature vessel formation. As the neovasculature matures, ECs form tightly regulated junctions to each other (Xu \& Cleaver, 2011). The ECs in our study may not yet have formed these tight boundaries leading to increased extracellular space between each cell and therefore causing the larger than expected diameters. The vessels formed also exhibit a slightly irregular morphology, and lacked a definitive circumscribed geometry. Vessel cross-sections typically yield lumens with an orbicular shape (Gao \& Drew, 2014). Whereas once again, this could be indicative of less mature vasculature that has yet to form a more robust geometry, these results are consistent with our previous studies (Grainger et al., 2013). Vessels formed by both types of ECs contained few, if any, erythrocytes at Day 4 within their lumens. Their presence at later time points suggests inosculation with the host vasculature occurred between Days 4 and 7. Qualitatively, extravascular erythrocytes decreased by Day 14, similar to a previous study (Grainger et al., 2013), suggesting stabilization and maturation of vessels. However, free erythrocytes still remained in the interstitial space of constructs containing iPSC-ECs, further supporting the aforementioned lack of vessel maturity.

Despite morphologic and phenotypic similarities in vessels formed from both EC types, IHC staining for hCD31 demonstrated quantitative differences in the vasculogenic abilities of iPSC-ECs. This may be due to reduced abilities of iPSC-ECs (relative to HUVECs) to proteolytically degrade the fibrin matrix, as they exhibit reduced expression of MMP-9 (Bezenah et al., 2018). However, while vessel density was significantly reduced at Days 4 and 7 , there were no significant differences between iPSC-ECs and HUVECs at later time points. Quantification of vessels containing erythrocytes further revealed vasculature formed by iPSC-ECs was less perfused. Early time points showed no difference between the two cell conditions, but the relatively low density of perfused vessels indicates the neovascualture formed did not sufficiently inosculate with the host's vasculature at this time. The large increase in vessel diameter by Day 7 suggests inosculation sometime between Days 4 and 7. We attributed the observed significant differences in vessel density (both total and perfused vessels) to the reduced abilities of iPSC-ECs to undergo capillary morphogenesis. By Day 14, densities of perfused vessels in implants containing either HUVECs or iPSC-ECs were similar due to significant reduction in vessel density for the HUVEC group. This regression in the HUVEC group is consistent with a previous study from our group (Grainger et al., 2013). Vessels undergo selective branch regression and pruning during the normal process of wound healing angiogenesis, changing network architecture over time as tissue metabolic demands change (Korn \& Augustin, 2015). This could be a result of increased tissue oxygenation by the HUVEC group at earlier time points, causing a downregulation of VEGF expression, a key signal involved in EC proliferation and invasion during vessel formation, and EC apoptosis in the newly established vessels (Simonavicius et al., 2012).

In addition to the observed differences in vessel quantity, vessels formed by the iPSC-ECs showed lower levels of COL-IV and $\alpha$-SMA staining, which may be indicative of a reduced maturity. COL-IV is a prominent component of the basement membrane of mature capillaries (Li, 2003), and thus the presence of this component is indicative of ECs' ability to form mature capillary networks. In addition, smooth muscle $\alpha$-actin is commonly used as a pericyte marker (Skalli et al., 1989), although not a very selective one as it also stains myofibroblasts (Hinz, 2007). Pericytes stabilize nascent vasculature and their presence suggests mature capillary networks (Ghajar et al., 2010; Skalli et al., 1989). As revealed by positive $\alpha$-SMA staining, the coimplanted NHLFs associated with the vessels formed in a pericyte-like manner. However, whether these NHLFs are capable of becoming bonafide pericytes in either EC condition is difficult to prove given the limited availability of bonafide pericyte markers (Bexell et al., 2009; Shi \& Gronthos, 2003). Although still significantly different compared with the HUVECs, iPSC-ECs demonstrated an increase in $\alpha$-SMA+ staining at Day 14 , perhaps indicating more recruitment of the NHLFs over time. Collectively, these data suggest iPSC-ECs are capable of recruiting and signaling stromal cells to differentiate into pericytes and depositing the components necessary for a basement membrane in vivo, but to a lesser degree than HUVECs.

Despite these differences in comparison to HUVECs, the iPSC-ECs performed better in vivo than expected relative to our previous in vitro findings (Bezenah et al., 2018). This perhaps suggests the in vivo microenvironment is able to induce the iPSC-ECs to attain a more mature phenotype. As the iPSCs from which the iPSC-ECs were differentiated were themselves generated from fibroblasts via an artificial reprogramming process, the iPSC-ECs have never been exposed to physiologic conditions, including blood flow. Shear stresses play a critical role in vascular development and EC phenotype (Rundek \& DellaMorte, 2015), and one avenue for future studies would be to explore the ability to induce maturation of the iPSC-ECs through exposure to fluid flow. In addition, while this experiment chose to use NHLFs as the stromal cells for coinjection, different stromal cells may better support iPSC-ECs vessel formation. Recent research has demonstrated differences in vascular morphogenesis of iPSC-ECs in vitro when cocultured with stromal cells of varying identities (Halaidych et al., 2018). Coinjecting different stromal cells may aid in the maturation of the iPSC-ECs and as a result, increase the number of vessels formed. Furthermore, while the subcutaneous implant model used here is relatively simple and effectively demonstrated differences in vessel formation, evaluating iPSC-ECs in more advanced in vivo models is critical to aid clinical translation. For example, a window-chamber model has enabled real-time visualization of vessel formation and anastomosis (G. Cheng et al., 2011) and may be useful to understand the abilities of iPSC-ECs to inosculate with host vessels. Similarly, hind limb ischemia models, where the lack of oxygen and nutrients may be more challenging (or more stimulatory) for vessel formation, have already been used to demonstrate the ability of iPSC-ECs to rescue ischemic tissue in a situation that better replicates the clinical target (Rufaihah et al., 2011). Our data here underscore the importance of comparing the efficacy of iPSC-ECs head-to-head with HUVECs (and other sources of ECs) in these more advanced models as well. 
In summary, this study assessed whether iPSC-ECs form functional microvasculature in a manner quantitatively and qualitatively similar to HUVECs in a well-characterized three-dimensional fibrin-based model of vasculogenesis in vivo. Both iPSC-ECs and HUVECs formed vessels with similar phenotypes and morphologies and demonstrated some characteristics of vessel maturation. However, the iPSC-ECs exhibited significantly reduced vessel density, perfusion, and maturation in comparison with HUVECs.

\section{ACKNOWLEDGMENTS}

This workstudy was partially supported by a grant from the National Institutes of Health (R01-HL085339). JRB was partially supported by a predoctoral fellowship from the NIH Cellular Biotechnology Training Grant (T32-GM008353) at the University of Michigan.

\section{ORCID}

Jonathan R. Bezenah (D) http://orcid.org/0000-0002-3271-2809

\section{REFERENCES}

Adams, W. J., Zhang, Y., Cloutier, J., Kuchimanchi, P., Newton, G., Sehrawat, S., ... García-Cardeña, G. (2013). Functional vascular endothelium derived from human induced pluripotent stem cells. Stem Cell Reports, 1(2), 105113. https://doi.org/10.1016/j.stemcr.2013.06.007

Allen, P., Melero-Martin, J., \& Bischoff, J. (2011). Type I collagen, fibrin, and puramatrix matrices provide permissive environments for human endothelial and mesenchymal progenitor cells to form neovascular networks. Journal of Tissue Engineering and Regenerative Medicine, 5(4), e74-e86. https://doi.org/10.1002/term.389

Au, P., Tam, J., Fukumura, D., \& Jain, R. K. (2008). Bone marrow-derived mesenchymal stem cells facilitate engineering of long-lasting functional vasculature. Blood, 111(9), 4551-4558. https://doi.org/10. 1182/blood-2007-10-118273

Bexell, D., Gunnarsson, S., Tormin, A., Darabi, A., Gisselsson, D., Roybon, L., ... Bengzon, J. (2009). Bone marrow multipotent mesenchymal stroma cells act as pericyte-like migratory vehicles in experimental gliomas. Molecular Therapy: The Journal of the American Society of Gene Therapy, 17(1), 183-190. https://doi.org/10.1038/mt.2008.229

Bezenah, J. R., Kong, Y. P., \& Putnam, A. J. (2018). Evaluating the potential of endothelial cells derived from human induced pluripotent stem cells to form microvascular networks in 3D cultures. Scientific Reports, 8, 2671. https://doi.org/10.1038/s41598-018-20966-1

Blache, U., \& Ehrbar, M. (2018). Inspired by nature: Hydrogels as versatile tools for vascular engineering. Advances in Wound Care, 7(7), 232-246. https://doi.org/10.1089/wound.2017.0760

Ceccarelli, J., \& Putnam, A. J. (2014). Sculpting the blank slate: How fibrin's support of vascularization can inspire biomaterial design. Acta Biomaterialia, 10(4), 1515-1523. https://doi.org/10.1016/j.actbio.2013.07.043

Chen, D. Y., Wei, H. J., Lin, K. J., Huang, C. C., Wang, C. C., \& Sung, H. W. (2004). Three-dimensional cell aggregates composed of HUVECs and cbMSCs for therapeutic neovascularization in a mouse model of hindlimb ischemia. Biomaterials, 34(8), 1995-2004. https://doi.org/10. 1016/j.biomaterials.2012.11.045

Chen, X., Aledia, A. S., Popson, S. A., Him, L., Hughes, C. C. W., \& George, S. C. (2010). Rapid anastomosis of endothelial progenitor cell-derived vessels with host vasculature is promoted by a high density of cotransplanted fibroblasts. Tissue Engineering. Part A, 16(2), 585-594. https://doi.org/10.1089/ten.tea.2009.0491
Cheng, G., Liao, S., Kit Wong, H., Lacorre, D. A., Di Tomaso, E., Au, P., ... Munn, L. L. (2011). Engineered blood vessel networks connect to host vasculature via wrapping-and-tapping anastomosis. Blood, 118(17), 4740-4749. https://doi.org/10.1182/blood-2011-02-338426

Christman, K. L., Vardanian, A. J., Fang, Q., Sievers, R. E., Fok, H. H., \& Lee, R. J. (2004). Injectable fibrin scaffold improves cell transplant survival, reduces infarct expansion, and induces neovasculature formation in ischemic myocardium. Journal of the American College of Cardiology, 44(3), 654-660. https://doi.org/10.1016/j.jacc.2004.04.040

Davies, M. G. (2012). Critical limb ischemia: Epidemiology. Methodist DeBakey Cardiovascular Journal, 8(4), 10-14.

Gao, Y.-R., \& Drew, P. J. (2014). Determination of vessel cross-sectional area by thresholding in radon space. Journal of Cerebral Blood Flow \& Metabolism, 34(7), 1180-1187. https://doi.org/10.1038/jcbfm. 2014.67

Ghajar, C. M., Blevins, K. S., Hughes, C. C. W., George, S. C., \& Putnam, A. J. (2006). Mesenchymal stem cells enhance angiogenesis in mechanically viable prevascularized tissues via early matrix metalloproteinase upregulation. Tissue Engineering, 12(10), 2875-2888. https://doi.org/ 10.1089/ten.2006.12.2875

Ghajar, C. M., Chen, X., Harris, J. W., Suresh, V., Hughes, C. C. W., Jeon, N. L., ... George, S. C. (2008). The effect of matrix density on the regulation of 3-D capillary morphogenesis. Biophysical Journal, 94(5), 1930-1941. https://doi.org/10.1529/biophysj.107.120774

Ghajar, C. M., Kachgal, S., Kniazeva, E., Mori, H., Costes, S. V., George, S. C., \& Putnam, A. J. (2010). Mesenchymal cells stimulate capillary morphogenesis via distinct proteolytic mechanisms. Experimental Cell Research, 316(5), 813-825. https://doi.org/10.1016/j.yexcr.2010.01.013

Grainger, S. J., Carrion, B., Ceccarelli, J., \& Putnam, A. J. (2013). Stromal cell identity influences the in vivo functionality of engineered capillary networks formed by co-delivery of endothelial cells and stromal cells. Tissue Engineering. Part A, 19(9-10), 1209-1222. https://doi.org/10. 1089/ten.tea.2012.0281

Halaidych, O. V., Freund, C., van den Hil, F., Salvatori, D. C. F., Riminucci, M., Mummery, C. L., \& Orlova, V. V. (2018). Inflammatory responses and barrier function of endothelial cells derived from human induced pluripotent stem cells. Stem Cell Reports, 10(5), 1642-1656. https:// doi.org/10.1016/j.stemcr.2018.03.012

Hinz, B. (2007). Formation and function of the myofibroblast during tissue repair. Journal of Investigative Dermatology, 127(3), 526-537. https:// doi.org/10.1038/sj.jid.5700613

Ikada, Y. (2006). Challenges in tissue engineering. Journal of the Royal Society Interface, 3(10), 589-601. https://doi.org/10.1098/rsif.2006.0124

Ikuno, T., Masumoto, H., Yamamizu, K., Yoshioka, M., Minakata, K., Ikeda, T., ... Yamashita, J. K. (2017). Efficient and robust differentiation of endothelial cells from human induced pluripotent stem cells via lineage control with VEGF and cyclic AMP. PLoS One, 12(3), e0173271. https://doi.org/10.1371/journal.pone.0173271

Kannan, R. Y., Salacinski, H. J., Sales, K., Butler, P., \& Seifalian, A. M. (2005). The roles of tissue engineering and vascularisation in the development of microvascular networks: A review. Biomaterials, 26(14), 1857-1875. https://doi.org/10.1016/j.biomaterials.2004.07.006

Kniazeva, E., Kachgal, S., \& Putnam, A. J. (2011). Effects of extracellular matrix density and mesenchymal stem cells on neovascularization in vivo. Tissue Engineering. Part A, 17(7-8), 905-914. https://doi.org/10. 1089/ten.tea.2010.0275

Koike, N., Fukumura, D., Gralla, O., Au, P., Schechner, J. S., \& Jain, R. K. (2004). Tissue engineering: Creation of long-lasting blood vessels. Nature, 428(6979), 138-139. https://doi.org/10.1038/428138a

Korn, C., \& Augustin, H. G. (2015). Mechanisms of vessel pruning and regression. Developmental Cell, 34(1), 5-17. https://doi.org/10.1016/j. devcel.2015.06.004

Li, A. C. Y., \& Thompson, R. P. H. (2003). Basement membrane components. Journal of Clinical Pathology, 56(12), 885-887. https:// doi.org/10.1136/jcp.56.12.885 
Margariti, A., Winkler, B., Karamariti, E., Zampetaki, A., Tsai, T., Baban, D., ... Xu, Q. (2012). Direct reprogramming of fibroblasts into endothelial cells capable of angiogenesis and reendothelialization in tissueengineered vessels. Proceedings of the National Academy of Sciences of the United States of America, 109(34), 13793-13798. https://doi.org/ 10.1073/pnas.1205526109

Melero-Martin, J. M., De Obaldia, M. E., Kang, S. Y., Khan, Z. A., Yuan, L., Oettgen, P., \& Bischoff, J. (2008). Engineering robust and functional vascular networks in vivo with human adult and cord blood-derived progenitor cells. Circulation Research, 103(2), 194-202. https://doi. org/10.1161/CIRCRESAHA.108.178590

Mozaffarian, D., Benjamin, E. J., Go, A. S., Arnett, D. K., Blaha, M. J., Cushman, M., ... Turner, M. B. (2015). AHA statistical update: Executive summary: Heart disease and stroke statistics-2015 update: A report from the American Heart Association. Circulation, 131, 434-441. https://doi.org/10.1161/CIR.0000000000000152

Peterson, A. W., Caldwell, D. J., Rioja, A. Y., Rao, R. R., Putnam, A. J., \& Stegemann, J. P. (2014). Vasculogenesis and angiogenesis in modular collagen-fibrin microtissues. Biomaterials Science, 2(10), 1497-1508. https://doi.org/10.1039/C4BM00141A

Potter, R. F., \& Groom, A. C. (1983). Capillary diameter and geometry in cardiac and skeletal muscle studied by means of corrosion casts. Microvasculature Research, 1, 68-84. https://doi.org/10.1016/00262862(83)90044-4

Rao, R. R., Peterson, A. W., Ceccarelli, J., Putnam, A. J., \& Stegemann, J. P. (2012). Matrix composition regulates three-dimensional network formation by endothelial cells and mesenchymal stem cells in collagen/fibrin materials. Angiogenesis, 15(2), 253-264. https://doi. org/10.1007/s10456-012-9257-1

Roger, V. L., Go, A. S., Lloyd-Jones, D. M., Adams, R. J., Berry, J. D., Brown, T. M., ... Wylie-Rosett, J. (2011). Heart disease and stroke statistics-2011 update: A report from the American Heart Association. Circulation, 123(4), e18-e209. https://doi.org/10.1161/CIR. Ob013e3182009701

Rouwkema, J., Rivron, N. C., \& van Blitterswijk, C. A. (2008). Vascularization in tissue engineering. Trends in Biotechnology, 26(8), 434-441. https://doi.org/10.1016/j.tibtech.2008.04.009

Rubina, K., Kalinina, N., Efimenko, A., Lopatina, T., Melikhova, V., Tsokolaeva, Z., ... Parfyonova, Y. (2009). Adipose stromal cells stimulate angiogenesis via promoting progenitor cell differentiation, secretion of angiogenic factors, and enhancing vessel maturation. Tissue Engineering. Part A, 15(8), 2039-2050. https://doi.org/10.1089/ ten.tea.2008.0359

Rufaihah, A. J., Huang, N. F., Jamé, S., Lee, J. C., Nguyen, H. N., Byers, B., ... Cooke, J. P. (2011). Endothelial cells derived from human iPSCS increase capillary density and improve perfusion in a mouse model of peripheral arterial disease. Arteriosclerosis, Thrombosis, and Vascular Biology, 31(11), e72-e79. https://doi.org/10.1161/ATVBAHA. 111.230938

Rufaihah, A. J., Huang, N. F., Kim, J., Herold, J., Volz, K. S., Park, T. S., ... Cooke, J. P. (2013). Human induced pluripotent stem cell-derived endothelial cells exhibit functional heterogeneity. American Journal of Translational Research, 5(1), 21-35. http://www.pubmedcentral.nih.gov/

Rundek, T., \& Della-Morte, D. (2015). The role of shear stress and arteriogenesis in maintaining vascular homeostasis and preventing cerebral atherosclerosis. Brain Circulation, 1(1), 53. https://doi.org/10. 4103/2394-8108.164993

Saigawa, T., Kato, K., Ozawa, T., Toba, K., Makiyama, Y., Minagawa, S., ... Aizawa, Y. (2004). Clinical application of bone marrow implantation in patients with arteriosclerosis obliterans, and the association between efficacy and the number of implanted bone marrow cells. Circulation Journal, 68(12), 1189-1193. https://doi.org/10.1253/circj.68.1189

Shi, S., \& Gronthos, S. (2003). Perivascular niche of postnatal mesenchymal stem cells in human bone marrow and dental pulp. Journal of
Bone and Mineral Research, 18(696), 696-704. https://doi.org/10. 1359/jbmr.2003.18.4.696

Simonavicius, N., Ashenden, M., van Weverwijk, A., Lax, S., Huso, D. L., Buckley, C. D., ... Isacke, C. M. (2012). Pericytes promote selective vessel regression to regulate vascular patterning. Blood, 120(7), 1516-1527. https://doi.org/10.1182/blood-2011-01-332338

Skalli, O., Pelte, M. F., Peclet, M. C., Gabbiani, G., Gugliotta, P., Bussolati, G., ... Orci, L. (1989). Alpha-smooth muscle actin, a differentiation marker of smooth muscle cells, is present in microfilamentous bundles of pericytes. Journal of Histochemistry \& Cytochemistry, 37(3), 315-321. https://doi.org/10.1177/37.3.2918221

Sun, Q., Silva, E. A., Wang, A., Fritton, J. C., Mooney, D. J., Schaffler, M. B., ... Rajagopalan, S. (2010). Sustained release of multiple growth factors from injectable polymeric system as a novel therapeutic approach towards angiogenesis. Pharmaceutical Research, 27(2), 264-271. https://doi.org/10.1007/s11095-009-0014-0

Takahashi, K., \& Yamanaka, S. (2006). Induction of pluripotent stem cells from mouse embryonic and adult fibroblast cultures by defined factors. Cell, 126(4), 663-676. https://doi.org/10.1016/j.cell.2006.07.024

Taleb, S. (2016). Inflammation in atherosclerosis. Archives of Cardiovascular Diseases, 9(12), 708-715. https://doi.org/10.1016/j.acvd.2016.04.002

Tarride, J. E., Lim, M., DesMeules, M., Luo, W., Burke, N., O’Reilly, D., ... Goeree, R. (2009). A review of the cost of cardiovascular disease. The Canadian Journal of Cardiology, 25(6), e195-e202. https://doi.org/10. 1016/S0828-282×(09)70098-4

Traktuev, D. O., Prater, D. N., Merfeld-Clauss, S., Sanjeevaiah, A. R., Saadatzadeh, M. R., \& March, K. L. (2009). Robust functional vascular network formation in vivo by cooperation of adipose progenitor and endothelial cells. Circulation Research, 104, 1410-1420. https://doi. org/10.1161/CIRCRESAHA.108.190926

Wiedeman, M. P. (1963). Dimensions of blood vessels from distributing artery to collecting vein. Circulation Research, 9, 375-378. https://doi. org/10.1161/01.RES.12.4.375

Wong, W. T., Sayed, N., \& Cooke, J. P. (2013). Induced pluripotent stem cells: How they will change the practice of cardiovascular medicine. Methodist DeBakey Cardiovascular Journal, 9(4), 206-209. https://doi. org/10.14797/mdcj-9-4-206

Xu, K., \& Cleaver, O. (2011). Tubulogenesis during blood vessel formation. Seminars in Cell \& Developmental Biology, 22(9), 993-1004. https://doi. org/10.1016/j.semcdb.2011.05.001

Yancopoulos, G. D., Davis, S., Gale, N. W., Rudge, J. S., Wiegand, S. J., \& Holash, J. (2000). Vascular-specific growth factors and blood vessel formation. Nature, 407(6801), 242-248. https://doi.org/10.1038/35025215

Yee, J. (2010). Turning somatic cells into pluripotent stem cells. Nature Education, 3(9), 25.

Yoder, M. C. (2015). Differentiation of pluripotent stem cells into endothelial cells. Current Opinion in Hematology, 22(3), 252-257. https://doi.org/10.1097/MOH.0000000000000140

Zhang, H., Zhang, N., Li, M., Feng, H., Jin, W., Zhao, H., ... Tian, L. (2008). Therapeutic angiogenesis of bone marrow mononuclear cells (MNCs) and peripheral blood MNCs: Transplantation for ischemic hindlimb. Annals of Vascular Surgery, 22(2), 238-247. https://doi.org/10.1016/j. avsg.2007.07.037

How to cite this article: Bezenah JR, Rioja AY, Juliar B, Friend $\mathrm{N}$, Putnam AJ. Assessing the ability of human endothelial cells derived from induced-pluripotent stem cells to form functional microvasculature in vivo. Biotechnology and Bioengineering. 2019;116:415-426. https://doi.org/10.1002/bit.26860 